Manuscript received October 15 , 2018; accepted for publication April 25, 2019; published online June 19, 2019.

1 Research Center E. Piaggio, University of Pisa, Largo Lucio Lazzarino 1, Pisa 56122, Italy, (D) https://orcid.org/0000-00025918-3695 (H.G.), (1D https://orcid. org/0000-0001-5697-6027

(A.D.A.), (1) https://orcid.org/ 0000-0003-4023-4111 (F.M.), (1) https://orcid.org/0000-00021368-3571 (C.D.M.)

2 Department of Information Engineering, University of Pisa, Largo Lucio Lazzarino 1, Pisa 56122, Italy

${ }^{3}$ Research Center E. Piaggio, University of Pisa, Largo Lucio Lazzarino 1, Pisa 56122, Italy; and Department of Information Engineering, University of Pisa, Largo Lucio Lazzarino 1, Pisa 56122, Italy (Corresponding author), e-mail: g.vozzi@ing.unipi.it, (1) https://orcid.org/0000-00029414-9994
Harish Gunasekaran, ${ }^{1}$ Aurora De Acutis, ${ }^{1,2}$ Francesca Montemurro, ${ }^{1,2}$ Carmelo De Maria, ${ }^{1,2}$ and Giovanni Vozzi ${ }^{3}$

\section{Fabrication and Characterization of Gelatin/Carbon Black-Based Scaffolds for Neural Tissue Engineering Applications}

\section{Reference}

H. Gunasekaran, A. De Acutis, F. Montemurro, C. De Maria, and G. Vozzi, "Fabrication and Characterization of Gelatin/Carbon Black-Based Scaffolds for Neural Tissue Engineering Applications," Materials Performance and Characterization 8, no. 1 (2019): 301-315. https:// doi.org/10.1520/MPC20180165

\section{ABSTRACT}

Neural tissue engineering has recently emerged as an alternative strategy to repair nerve damage and promote nerve regeneration. It involves the fabrication of scaffolds with properties mimicking those of the natural extracellular matrix for guiding a three-dimensional (3D) neural regeneration. These engineered constructs, in addition to mechanical support, should be also capable of providing proper chemical and electrical stimuli for adhesion, migration, and proliferation of the neural cells. In this study, we developed conductive composite hydrogel films based on gelatin and carbon black (CB) as scaffolds for neural tissue engineering applications. The presented hydrogel constructs were fabricated in the form of films using the solvent casting method after dispersing several concentrations of $\mathrm{CB}$ in a $5 \%(\mathrm{w} / \mathrm{v})$ gelatin solution along with (3-glycidoxypropyl) trimethoxysilane (GPTMS) as the crosslinking agent at a concentration of $1.84 \%(\mathrm{v} / \mathrm{v})$. The CB concentrations of $0.3 \%, 0.5 \%, 0.7 \%$, and $0.9 \%(\mathrm{w} / \mathrm{w})$ with respect to the gelatin amount were chosen. The morphological, compositional, swelling, dissolution, electrical, mechanical, and wettability properties together were characterized as function of CB content and compared with those of pure gelatin-based hydrogel. Results demonstrated that the incorporation of different quantities of $\mathrm{CB}$ relatively reduced the water uptake capability of the films and increased the stability in water of the gelatin matrix. Findings from the mechanical tests revealed that composite hydrogels have a lower elastic modulus with respect to the pure gelatin matrix. Moreover, it was found that the incorporation of incremental CB concentrations kept the wettability surface property unchanged while the electrical characterization of the proposed structures showed a reduction of the electrical impedance. Overall, the study suggests that the composite structures could be used as a potential candidate for fabrication of scaffolds for neural regeneration with tunable electrical and mechanical properties by varying the CB concentration in a finite range. 


\section{Keywords}

neural tissue engineering, neural scaffolds, gelatin-carbon black composite

\section{Introduction}

Every year, central and peripheral nerve injuries affect millions of people worldwide because of traumas, pathological events, or aging. These injuries are the most important cause of physical disability among patients, resulting also in enormous social and economic concerns. ${ }^{1}$ The repairing process in the nervous system is complicated to be clinically addressed. Despite the intrinsic regenerative capacity of the peripheral nervous system (PNS), nerve allograft or autograft represents the only therapeutic option for the improvement of neurological deficits even despite their drawbacks, such as lack of donors, neuroma, infection diseases, recurring pain, and sensory loss. ${ }^{2-3}$ Unlike in PNS, neuroregeneration in the central nervous system (CNS) is not possible under the normal conditions because several factors can cause its failure, consequently, any clinical management of CNS injuries is still not promising. ${ }^{4}$

Recent efforts in regenerative medicine, especially in the tissue engineering field, have been made with the aim to develop an engineering approach for the regeneration, repair, and replacement of the human PNS and CNS tissues at the site of injury. ${ }^{5}$ The tissue engineering approach is generally based on the triad, consisting of scaffolds, cells, and growth-stimulating signals, which helps to develop a biological substitute to replace the diseased tissues. ${ }^{6}$

Scaffolds are bioactive and biodegradable engineered structures, typically made of polymeric biomaterials, whose function is to provide a structural support for cell attachment so as to guide the tissue regeneration in three dimensions. The ideal scaffold for an engineered tissue should match the extracellular matrix (ECM) of the target tissue from the topological, chemical, biological, and mechanical points of view to mimic the physiological microenvironment that controls specific cellular responses. ${ }^{6}$ Many studies on nerve cells have revealed that the electrical signals also play pivotal roles for controlling cell metabolic activity, cell adhesion, proliferation, and differentiation. ${ }^{7}$ Therefore, for the neural tissue engineering purposes, an important aspect of well-designed scaffolds for nerve regeneration is their ability to conduct electricity, thereby reinstating the electrical functionality of the neural tissue.

Electrically conductive polymers (CPs), such as polypyrrole (PPy), polyaniline (PANI), or poly(3,4-ethylenedioxythiophene) (PEDOT), have been widely used to fabricate neural tissue engineering scaffold because of their good electrical properties and processability. ${ }^{8-10}$ However, the biocompatibility of CPs has not been conclusively proved until now, and they are generally stiff and display a high hydrophobic nature. ${ }^{11,12}$ Thus, research efforts have been directed toward the fabrication of scaffolds using electroconductive and electroactive hydrogels, which intrinsically provide the biomechanical, electrical, and hydration properties akin to the native soft tissues. ${ }^{13}$ Conductive hydrogels can be generally fabricated by incorporating CPs or carbon-based nanofillers such as graphene, carbon nanotubes (CNTs) or carbon black (CB) into a synthetic or natural (or a combination of both) polymeric 3D aqueous matrix. ${ }^{12}$

Recently, natural polymers-based hydrogels have attracted attention as a bulk material for conductive scaffolds, because they show many desirable characteristics such as biocompatibility, biodegradability, and nontoxicity. ${ }^{14}$ Among all the natural biopolymers, gelatin, which is a biocompatible protein derived by acid or alkaline hydrolysis of collagen, is deemed very suitable for the tissue engineering applications thanks to its low antigenicity, cells adhesion, and migration-promoting characteristics. ${ }^{15}$

In the current study, novel conductive gelatin-based hydrogel scaffolds for neural tissue engineering purpose were fabricated by solvent casting technique in form of films. The conductive gelatin solution was prepared by introducing different quantities of a highly conductive $\mathrm{CB}$ powder into the $3 \mathrm{D}$ gelatin matrix. With a future prospective view to perform in vitro tests on the proposed structures, the gelatin matrix was chemically cross-linked to improve its mechanical properties and durability in aqueous media. To this purpose, in this work (3-Glycidoxypropyl)trimethoxysilane (GPTMS) was used as chemical crosslinker, given its capability to functionalize the gelatin matrix favoring cells adhesion and proliferation when added in an appropriate quantity to the 
gelatin solution. ${ }^{16,17}$ Among all the carbon-based additives, in this work, CB microparticles were selected to enhance the electrical properties of gelatin because of their numerous desirable properties, such as low cytotoxicity, good electrical conductivity, low cost, and good thermal and chemical stability. ${ }^{18,19}$

Herein, an investigation was carried out on the effects of the different $\mathrm{CB}$ concentrations on the morphological, compositional, electrical, mechanical, and swelling properties of the proposed hydrogels in order to explore their feasibility to be employed as scaffolds for future and tailored applications in the field of neural tissue repair.

\section{Experimental}

\section{MATERIALS}

Gelatin (Type A from porcine skin), GPTMS, and Tween 20 were purchased from Sigma Aldrich. CB powder (Vulcan XC-72) was supplied by Cabot Corporation.

\section{METHODS}

\section{Preparation of gelatin-CB-GPTMS Film Scaffolds}

The preparation of the gelatin-CB-GPTMS (G-CB-GPTMS) scaffolds by solvent casting technique is schematically shown in figure 1 . Firstly, a $5 \%(\mathrm{w} / \mathrm{v})$ gelatin solution was prepared by dissolving the gelatin powder in the Milli-Q ultrapure water at $50^{\circ} \mathrm{C}$ under continuous stirring on a magnetic stirrer. The $\mathrm{CB}$ suspension was prepared by dissolving the $\mathrm{CB}$ powder in Milli-Q water with a concentration of $1 \%(\mathrm{w} / \mathrm{v})$. After adding a volume of $100 \mu \mathrm{L}$ of Tween 20 to the suspension, a homogenous conductive solution was obtained by dispersing CB with sonication at a power of $2 \mathrm{~W}$ for $2-3 \mathrm{~h}$ ( $5 \mathrm{~min}$ break for every $15 \mathrm{~min}$ ).

The G-CB-GPTMS solutions with different $\mathrm{CB}$ amounts were obtained by adding to a volume of gelatin solution the $\mathrm{CB}$ solution at four concentrations of $0.3 \%, 0.5 \%, 0.7 \%$, and $0.9 \%(\mathrm{w} / \mathrm{w})$ with respect to gelatin solution along with GPTMS at concentration of $1.84 \%(\mathrm{v} / \mathrm{v})$. The amount of the crosslinker was selected on the basis of our previous works to guarantee the biocompatibility of the composite structures in view of the intended application. ${ }^{20}$ The resulting mixtures were then subjected to another sonication cycle for about $15 \mathrm{~min}$ at $2 \mathrm{~W}$ for obtaining a homogeneous G-CB-GPTMS solution.

FIG. 1 Schematic illustration of the fabrication process of G-CB-GPTMS scaffolds.

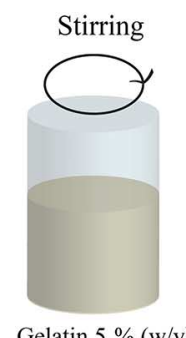

Gelatin $5 \%(w / v)$
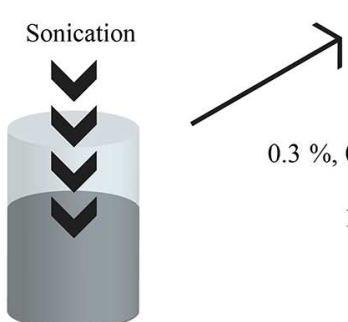

Gelatin

Casting in a Petri dish
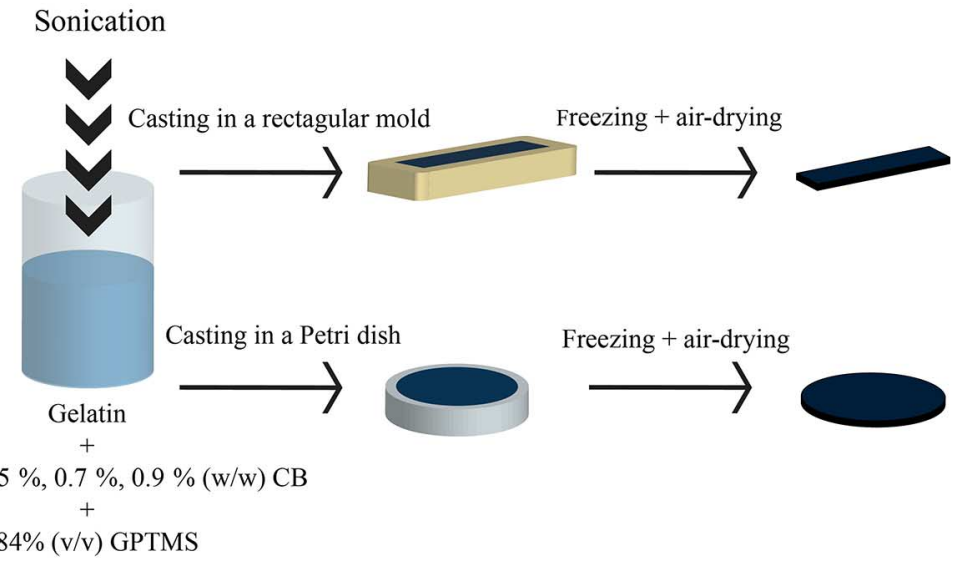

Freezing + air-drying

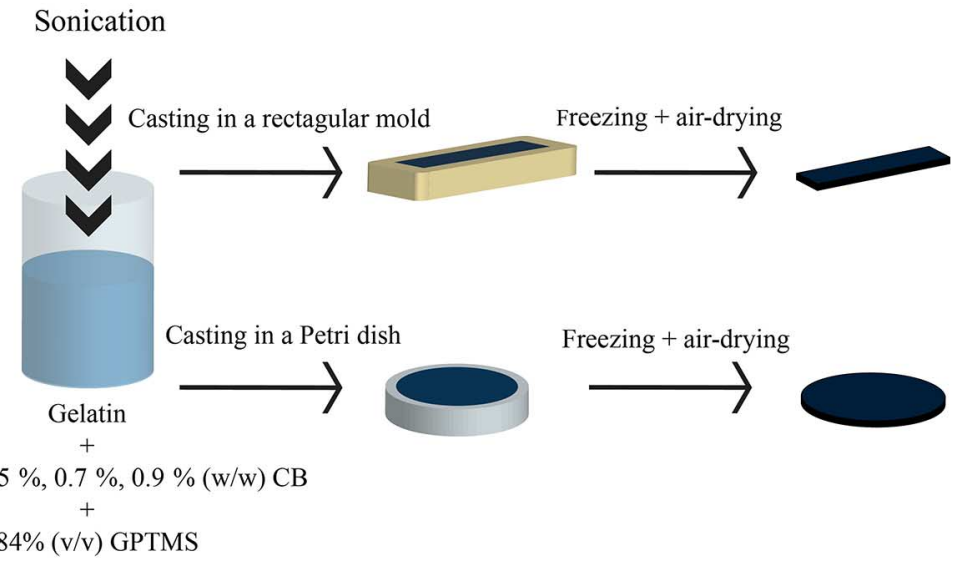

CB $1 \%(w / v)$ 
Starting from the G-CB-GPTMS solutions, the scaffolds were obtained by film casting technique according to procedures schematically described in figure 1. For the purpose of mechanical and swelling characterization, $2 \mathrm{~mL}$ of each G-CB-GPTMS solution were cast into a rectangular acrylic homemade mold (50 by 10 by $5 \mathrm{~mm}^{3}$ ) placed on a leveled table and frozen for $30 \mathrm{~min}$ to speed up the hydrogel formation and to avoid the CB precipitation. Then, samples were air-dried at a room temperature $\left(25^{\circ} \mathrm{C}\right)$ and demolded after $36 \mathrm{~h}$ casting to obtain rectangular dry thin films with an average thickness of $140 \mu \mathrm{m}$ (see fig. 2). For the purpose of electrical characterization, $4 \mathrm{~mL}$ of each G-CB-GPTMS solution were casted into polystyrene Petri dishes of $35 \mathrm{~mm}$ in diameter. With the same procedure, the control group films were prepared by casting gelatin-GPTMS solution (G-GPTMS, without any addition of $\mathrm{CB}$ ) in dedicated molds.

\section{Swelling and Dissolution Tests}

The procedure to evaluate water swelling properties of the G-CB-GPTMS hydrogel scaffolds can be summarized as follows: the weight of dry G-CB-GPTMS scaffolds was initially measured for each CB concentration under investigation. These scaffolds were then placed in Milli-Q water at $25^{\circ} \mathrm{C}$, and the weights of the hydrated samples were recorded at regular time intervals until they swelled to equilibrium. The mass swelling ratio (SR) was calculated as described by others ${ }^{21}$ :

$$
S R(\%)=\left(\frac{W s-W d}{W d}\right) \times 100
$$

where $W_{s}$ and $W_{d}$ are the swollen and dry weight, respectively.

At each time point (every $30 \mathrm{~min}$ for about $7 \mathrm{~h}$ ), the measurements were performed in triplicate on each kind of G-CB-GPTMS films and on control samples. After the swelling test, all the samples were dried at $25^{\circ} \mathrm{C}$ for $48 \mathrm{~h}$, and then they were weighted again and to estimate the dissolution percentage (D) as described $\mathrm{in}^{21}$ :

$$
D(\%)=\left(\frac{W d-W D}{W d}\right) \times 100
$$

where $W_{D}$ is the weight of the dried samples after the swelling test.

Moreover, the thickness of the rectangular dry and swelled films was evaluated using an analogic micrometer with an accuracy of $10 \mu \mathrm{m}$ and compared. In order to estimate the average thickness on each sample, three random locations on a film were used. The measurements were performed on three G-CB-GPTMS samples for each CB concentration under investigation and on three control samples.

\section{Contact Angle Measurements}

The water contact angle was measured using the sessile drop method. A Milli-Q water droplet of approximately $1 \mu \mathrm{L}$ was gently put in contact with the dry film surface. The profile of the sessile drop was then analyzed by means of a telescope-goniometer for calculating the contact angle according to the $\theta / 2 \operatorname{method}^{22}$ :

FIG. 2 Photograph and schematics of two examples of gelatin-based hydrogels prepared with two of the five concentrations (\% w/w) of CB under investigation. Gelatin without any addition of CB (control sample) or incorporating the maximum amount of $\mathrm{CB}$ are shown in the figure.

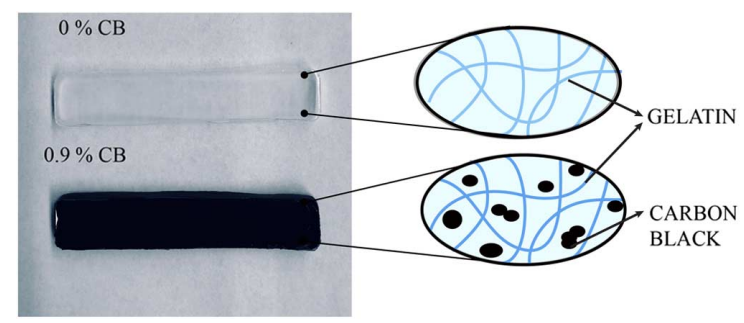




$$
\theta(\text { degree })=2 \tan ^{-1}\left(\frac{2 h}{w}\right)
$$

where $h$ is the height of the drop, and $w$ is the width of the drop. For the determination of the average contact angle on each sample, four readings were taken on random locations on the film. Each reported contact angle was the mean value of the measurements taken on three G-CB-GPTMS specimens for each CB concentration and on three control samples.

\section{Mechanical Characterization}

Mechanical properties of the rectangular-shaped hydrogel scaffolds were assessed by uniaxial tensile tests conducted on a Z005 series Zwick/Roell testing machine endowed with a 100-N load cell. The samples were hydrated prior to mechanical testing in Milli-Q water at $25^{\circ} \mathrm{C}$ for $2 \mathrm{~h}$. Each hydrated sample was fixed on the machine's standard grips covered with fine paper to reduce the slippage and then strained at a strain rate of $1 \%$ per minute until failure. From the stress-strain graph of each specimen, the tensile strength and the Young's modulus or elastic modulus $(E)$ were calculated. E was calculated as the slope of the fitted line on the stress-strain curve in the range of $3 \%$ to $5 \%$ of strain. Three G-CB-GPTMS specimens for each CB concentration and three control samples were tested and compared.

\section{Fourier Transform Infrared-Attenuated Total Reflectance Spectroscopy Analysis}

Fourier transform infrared (FTIR) spectroscopic analysis was performed on each single samples' component (G, CB, and GPTMS) and on both control (G-GPTMS) and test specimens (G-CB-GPTMS) in the range of $900-4,000 \mathrm{~cm}^{-1}$, using an Agilent 660 FTIR spectrophotometer with a focal plane array detector, operating in the Attenuated total reflection (ATR) mode. For each sample, data were acquired at a spectral resolution of a $4 \mathrm{~cm}^{-1}$ with 38 scans.

\section{Morphological Characterization}

Scanning electron microscope (SEM) images obtained using the Philips XL 20 were used to assess the crosssectional morphology of G-GPTMS and G-CB-GPTMS samples. Specimens were fractured along a direction parallel to the surface in liquid nitrogen using a cutting nipper. Samples were gold sputter-coated prior to SEM analysis. An acceleration voltage of $15 \mathrm{kV}$ and a working distance of about $10 \mathrm{~mm}$ were used to acquire images.

\section{Electrical Impedance Characterization}

Impedance measurements were performed using an Agilent E4980 impendence meter and a four-point probes configuration over a range of $20 \mathrm{~Hz}$ and $2 \mathrm{MHz}$ at $25^{\circ} \mathrm{C}$. Two adhesive copper electrodes were used as probeshydrogels interface. Measurements on round shaped scaffold were conducted on hydrated samples (after 2 hours of immersion in Milli-Q water) within Petri dishes. The experimental setup is shown in figure 3. Electrical impedance tests were performed in triplicate both on control samples (G-GPTMS) and on test samples (G-CB-GPTMS) at each $\mathrm{CB}$ concentration under investigation.

\section{Statistical Analysis}

Unless specified otherwise, all the results were presented as mean and standard deviation. One-way statistical analyses of variance combined with a Tukey's post hoc test were used with a significant level $p$ of 0.05 to determine differences among the samples.

\section{Results and Discussion}

\section{SWELLING AND DEGRADATION TESTS}

SR is one of the main properties that can affect biomaterials' biocompatibility. In fact, for the tissue engineering applications, a high degree of SR is a significant indicator of hydrogel scaffolds' capability to absorb water so as to 
FIG. 3

Picture of the

experimental setup used

for the electrical

impedance

measurement

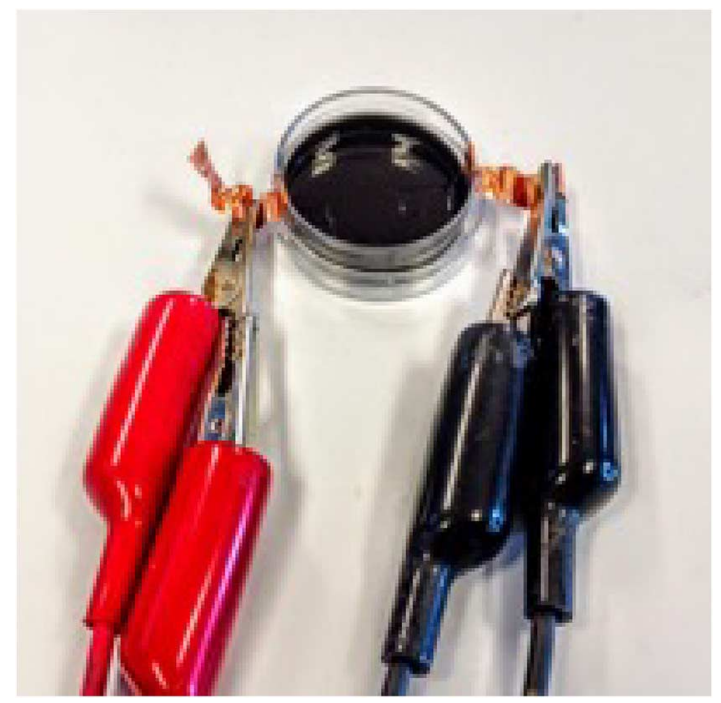

mimic the biomechanical properties of native tissues, to transfer nutrients and metabolites, and to promote cell infiltration and adhesion into the structure. The SRs of the G-CB-GPTMS samples and of the neat cross-linked gelatin samples were calculated according to equation (1), and the swelling profiles over time are shown in the graph in figure 4, with the standard deviations were marked using the error bars to give a clue about the statistical differences among the acquired data sets. It is seen that G-CB-GPTMS and G-GPTMS hydrogels had the same swelling trend even if, especially at the first time points of investigation, G-GPTMS scaffolds showed a higher SR than that of the samples filled with CB because of the completely hydrophilic nature of their bulk material. This means that water absorption and diffusion into the gel was hindered by the hydrophobic nature of the $\mathrm{CB}$ microparticles dispersed within the bulk materials. ${ }^{23,24}$ It was also found that the water uptake of the films decreased as CB content increased in the range $0.3-0.7 \%(w / w)$. This suggests that these concentrations allowed a homogeneous dispersion of the conductive filler within the matrix, and consequently, a homogenous crosslinking density between gelatin and CB particles, which hampered the diffusion of water molecules. ${ }^{25}$ Indeed, this behavior was not observed in the samples filled with the $0.9 \%$ of $\mathrm{CB}$, suggesting that this concentration did not guarantee a good dispersion of the filler in the matrix, but it induced the formation of CB agglomerations, which in turn reduced G-CB interactions so that to facilitate the diffusion of water within the hydrophilic matrix. However,

FIG. 4 Swelling property characterization. Percentage of increase of the weight (SR (\%)) of G-CB-GPTMS hydrogels as a function of time and $\mathrm{CB}$ concentrations obtained from the samples immersed in Milli-Q ultrapure water $\left(25^{\circ} \mathrm{C}\right)$ over time.

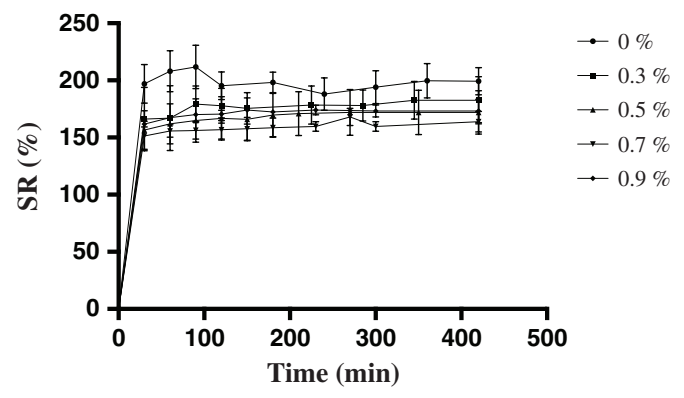


no statistical differences were found in the water uptake capability of G-GPTMS and all the G-CB-GPTMS samples just from the second hour of immersion in the aqueous medium (see fig. 5).

Given the shape of the sample, which can be approximated to a truss (i.e., with its length much more longer with respect to the other two dimensions) with a rectangular cross section, experimentally it was easier to measure the changes in length with respect to the changes in thickness, because of the isotropy of this phenomenon in these types of hydrogel. However, as the thickness of a construct for tissue engineering applications is a design parameter that can influence its mechanical and transport properties and the achievable cell density through the structure, ${ }^{26}$ the thickness of dry and hydrated samples was measured and compared in order to investigate how it could be influenced by different concentrations of CB. The measurements were performed after placing the samples in Milli-Q ultrapure water for $2 \mathrm{~h}$.

The average thickness of dry films of both G-CB-GPTMS and G-GPTMS was $140 \mu \mathrm{m}$ because the volume of solution used for solvent casting was always the same. The thickness of hydrated samples is reported in Table 1. No significant differences were found among the hydrated composite films and between composite films and controls; all the samples increased their thickness to about $70 \%$ with respect to their dry state. These findings suggested the addition of different amounts of $\mathrm{CB}$ could be embedded in the gelatin matrix without any impact on the wet films' thickness.

Finally, as regard to the degradation tests, after $7 \mathrm{~h}$ of immersion in Milli-Q water, the samples were dried to quantify their stability by measuring the weight loss according to equation (2). As shown in figure 6 , the percentage of degradation or loss of bulk material is significantly reduced as we increased CB concentrations. That is, the incorporation of $\mathrm{CB}$ in a concentration greater than $0.3 \%(\mathrm{w} / \mathrm{w})$ significantly increased the overall stability in the selected medium of the composite films with respect to the controls. However, no significant difference among all the G-CB-GPTMS films was found regarding their degradation.

FIG. 5

Swelling test results. SR of G-CB-GPTMS

hydrogels as a function of $\mathrm{CB}$ concentrations in the most significant time points.

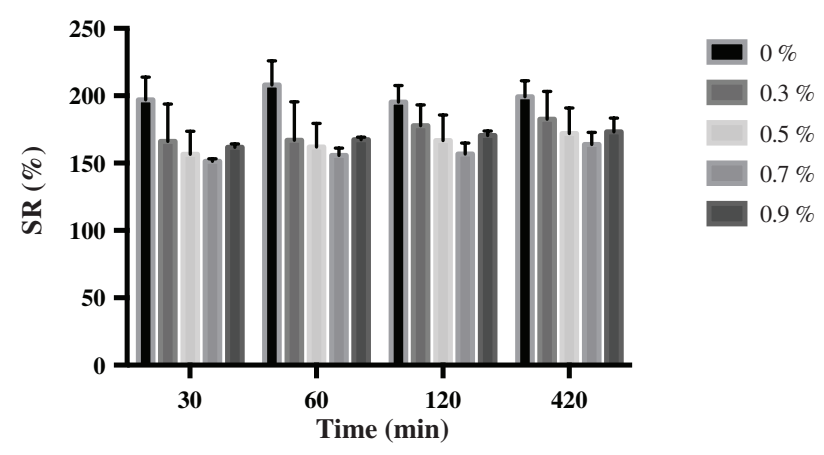

TABLE 1

G-CB-GPTMS film thickness as function of the CB content. The measurements were performed after immersing the samples for $2 \mathrm{~h}$ in Milli- $\mathrm{Q}$ ultrapure water $\left(25^{\circ} \mathrm{C}\right)$. No significant differences $(p<0.05)$ were found among each group

\begin{tabular}{lc}
\hline CB Content $(\% \mathrm{w} / \mathrm{w})$ & Film Thickness $(\mu \mathrm{m})$ \\
\hline 0 & $240.7 \pm 0.5$ \\
0.3 & $241.3 \pm 1.1$ \\
0.5 & $243.7 \pm 3.2$ \\
0.7 & $240.3 \pm 0.6$ \\
0.9 & $241 \pm 1.7$ \\
\hline
\end{tabular}


FIG. 6 Degradation characterization. Percentage decrease of the weight (D (\%)) of G-CB-GPTMS hydrogels for different CB concentrations. The measurements were performed on dried samples after about $7 \mathrm{~h}$ of immersion in Milli-Q ultrapure water. The symbol $\left(^{*}\right)$ indicates a statistical significance $(p<0.05)$ between the groups.

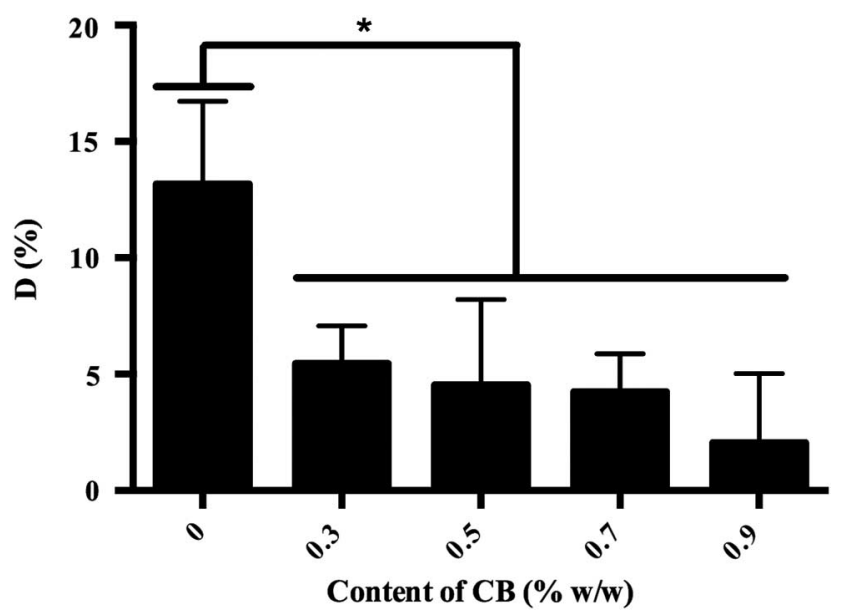

\section{MECHANICAL CHARACTERIZATION}

Because it is well known that the mechanical properties of scaffolds can significantly affect the behavior of cells, G-GPTMS and G-CB-GPTMS samples were mechanically tested to investigate the suitability of the proposed structures for neural regeneration. ${ }^{27,28}$ The Young's modulus $(E)$ was calculated from the stress-strain curves, and it is shown in figure 7 as a function of conductive filler content. In general, it is expected that a carbon-based additive contributes to the reinforcement of a polymeric matrix, and thus, an increase in $E$ is expected because of the addition of CB. In our study, on the contrary, it was found that the G-GPTMS samples showed a significantly higher $E$ when compared with the composites of G-CB-GPTMS samples. This behavior is in line with that described by other authors in literature. ${ }^{29}$ With the $0.3 \%$ (w/w) of CB, E decreased up to about $50 \%$ when compared with G-GPTMS samples, then it slightly increased when the conductive filler was increased until $0.7 \%(\mathrm{CB})$, and finally, with $0.9 \%$ (w/w) of $\mathrm{CB}$, a slight drop of $E$ was evidenced. However, comparing the elastic moduli of all the G-CB-GPTMS samples, no significance differences were observed among them. The results obtained in this section were in good accordance with previous studies about the effect of SR on the elastic modulus in hydrogels. ${ }^{30,31}$ In fact, the lower $E$ in the composite films with respect to pure G-GPTMS specimens was due to decreasing of the

FIG. 7

Mechanical

characterization of

G-CB-GPTMS hydrogel.

Young's moduli of the

composite gelatin-based

films for different

CB concentrations.

The symbol $\left({ }^{*}\right)$ indicates

a statistical significance

$(p<0.05)$ between the groups.

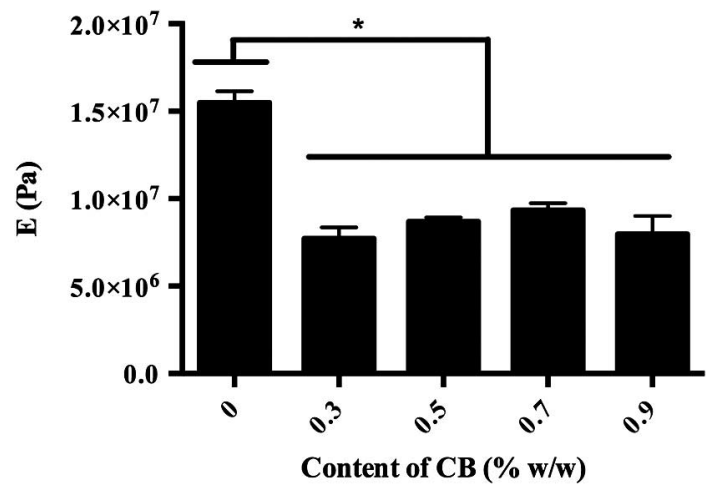


water uptake resulted mainly from the barrier effect of $\mathrm{CB}$ microparticles for the diffusion of water and its effect on the chemical crosslinking process between gelatin chains. ${ }^{30-32}$ Our experiments found that the incorporation of CB with a concentration between $0.3-0.7 \%(w / w)$ caused a growing homogeneous saturation of the gelatin matrix with hydrophobic conductive microparticles, thereby decreasing the SRs and increasing the elastic moduli (lower however respect to the pure G-GPTMS samples). The addition of $0.9 \%$ (w/w) of CB probably caused agglomerations of conductive microparticles that hampered gelatins chains interactions resulting in a higher water uptake accompanied by a drop of the elastic modulus. Moreover, our results suggested that the proposed G-CBGPTMS films reached its optimal concentration limit at $0.7 \%(\mathrm{w} / \mathrm{w})$ of conductive filler and in accordance with the percolation threshold theory previously reported by others. ${ }^{33}$

Results from the mechanical characterization also suggested that, even if the addition of the $\mathrm{CB}$ caused a significant lowering of elastic modulus with respect to the control samples, the G-CB-GPTM films did not achieve a suitable mechanical similarity with their natural counterparts. In fact, neural substrates have an elastic modulus approximately ranging from $2 \mathrm{kPa}$ to $40 \mathrm{kPa}^{34}$ Despite this aspect, it cannot be ruled out that the presented G-CB-GPTMS structure could support neural growth as proved by others in literature. ${ }^{35}$ In this regard, as a second stage of this work, the response of neural cells to these structures will be evaluated to validate whether there is an effect of interest to pursue this material for its final application or if a modulation of the composition of the structures will be needed in favor of structures with improved mechanical properties.

\section{MORPHOLOGICAL CHARACTERIZATION}

SEM was used to analyze the morphology of the proposed hydrogels. SEM micrographs of films' cross section at two high magnification $(\times 10,000$ and $\times 30,000)$ for each $\mathrm{CB}$ concentration under investigation are observed in figure 8. Morphological changes were detected inside the films because of the increasing amount of CB loading. As expected, the cross-sectional surface of pure cross-linked gelatin films appeared to be smooth, compact, and homogeneous. The cross-sectional surface of the films filled with the $0.3 \%$ and $0.5 \% \mathrm{CB}$ appeared compact and homogeneous as well, reflecting a good dispersion of the conductive microparticles within the cross-linked matrix. A loss of cross-sectional homogeneity was found in G-CB-GPTM films loaded with $0.7 \%$ of CB, in which the conductive microparticles appeared mostly well dispersed, even if some small CB aggregates started to form. Finally, the SEM images of G-CB-GPTMS films loaded with the $0.9 \%$ of CB showed a smooth cross section as those of pure cross-linked gelatin but endowed with $\mathrm{CB}$ agglomerations that demonstrated the absence of a uniform distribution of the conductive microparticles within the material. These findings confirmed all the deductions about the mechanical and swelling behavior of the presented G-CB-GPTMS hydrogels.

\section{CONTACT ANGLE MEASUREMENTS}

An important surface property that significantly influences the biocompatibility of a material for tissue engineering purposes is its surface wettability, which quantifies the capability of a fluid to spread on a surface. The wettability of a material, with respect to a specific fluid, can be determined by measuring the contact angle of the fluid and the material. ${ }^{36}$ In our study, we measured the value of water contact angle to investigate how the well-known hydrophilic properties of G-GPTMS films can be altered by the introduction of different contents of CB. The water contact angles were calculated according to equation (3), and the results are shown in figure 9: the hydrophilicity of the hydrogel films did not significantly change with the CB content and with respect to the control samples. This finding evidenced from the wettability point of view about the potentiality of the proposed hydrogel films to accommodate and support the neural cells' growth.

\section{FTIR-ATTENUATED TOTAL REFLECTANCE SPECTROSCOPY ANALYSIS}

FTIR-ATR spectra for CB, GPTMS, and pure gelatin are shown in figure 10. In the GPTMS's spectra, the broad peak at $1,087 \mathrm{~cm}^{-1}$ was characteristic of the $\mathrm{Si}-\mathrm{O}-\mathrm{CH}_{3}$ stretching vibration of ethoxy groups. The bands at $1,342 \mathrm{~cm}^{-1}$ and $1,640 \mathrm{~cm}^{-1}$ corresponded to the epoxy-ring stretching. Furthermore, peaks at 2,842 and $2,947 \mathrm{~cm}^{-1}$ accounted for $-\mathrm{CH}_{2}$ and $-\mathrm{CH}_{3}$ symmetric stretching. ${ }^{37}$ 
FIG. 8

Morphological

characterization of G-CB-GPTMS

hydrogels via SEM as a function of the

$\mathrm{CB}$ concentration at two magnifications.

\section{$\times 10 \mathrm{k}$}
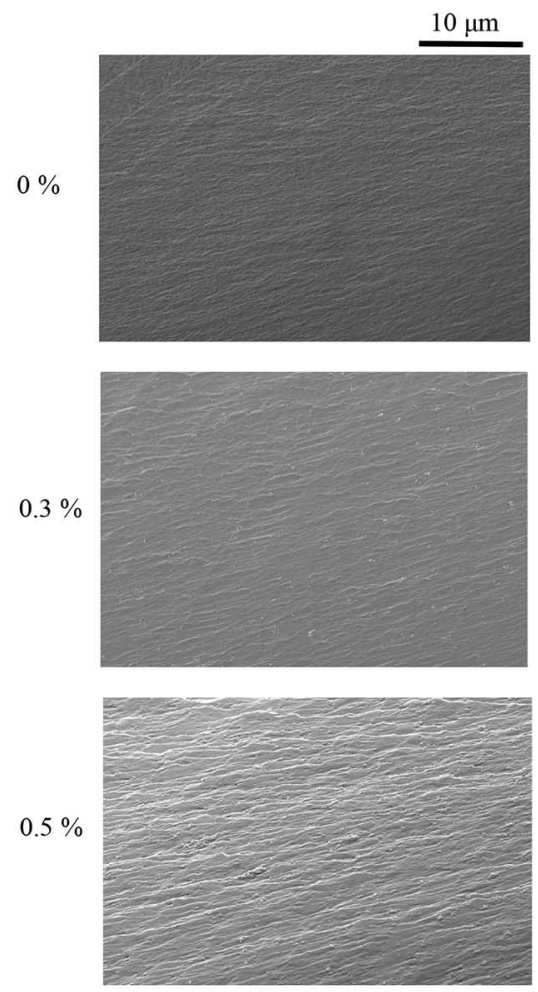

$0.7 \%$

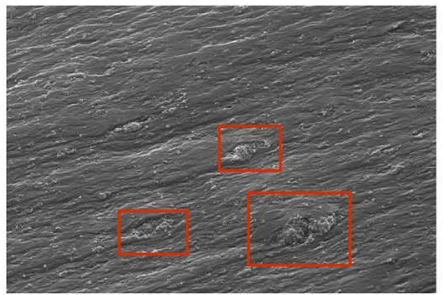

$0.9 \%$

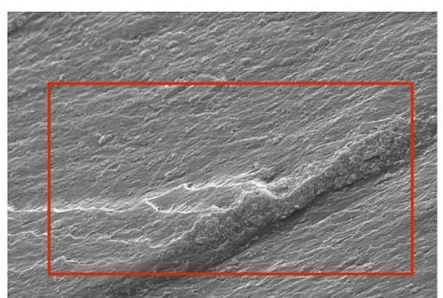

\section{$\times 30 \mathrm{k}$}
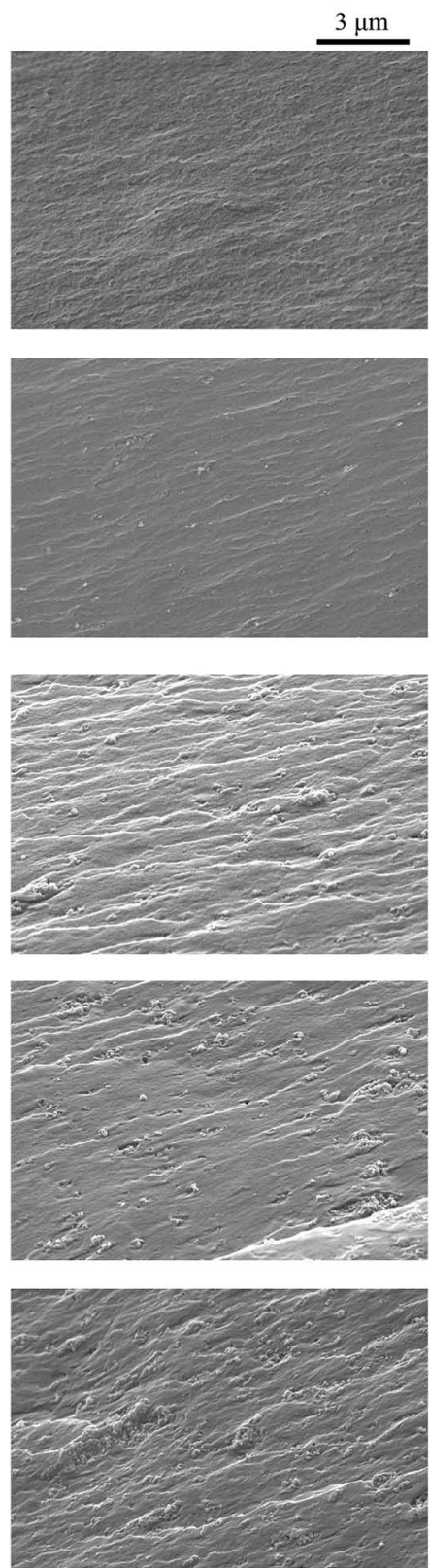

In the pure gelatin spectra, the bands at $1,239 \mathrm{~cm}^{-1}, 1,547 \mathrm{~cm}^{-1}, 1,630 \mathrm{~cm}^{-1}$, and $3,282 \mathrm{~cm}^{-1}$ were respectively related to the amide-A, amide-I, amide-II, and amide-III. Meanwhile, the bands at $3,293 \mathrm{~cm}^{-1}$ corresponded to the $\mathrm{N}-\mathrm{H}$ and $\mathrm{O}-\mathrm{H}$ stretching vibrations. ${ }^{38}$

In figure 11, FTIR spectra of G-CB-GPTMS samples are reported for every CB concentration under investigation. All the spectra clearly showed the peaks related to the gelatin's main functional groups. Additionally, characteristic bands of $\mathrm{Si}-\mathrm{O}-\mathrm{Si}$ bonds and of $\mathrm{Si}-\mathrm{OH}$ stretching vibrations were observed in all the spectra at 
FIG. 9

Characterization of the wettability surface property of the G-CBGPTMS films. Water contact angle was measured on hydrogels' surface for different CB concentrations. No significant differences $(p<0.05)$ were found among each group.

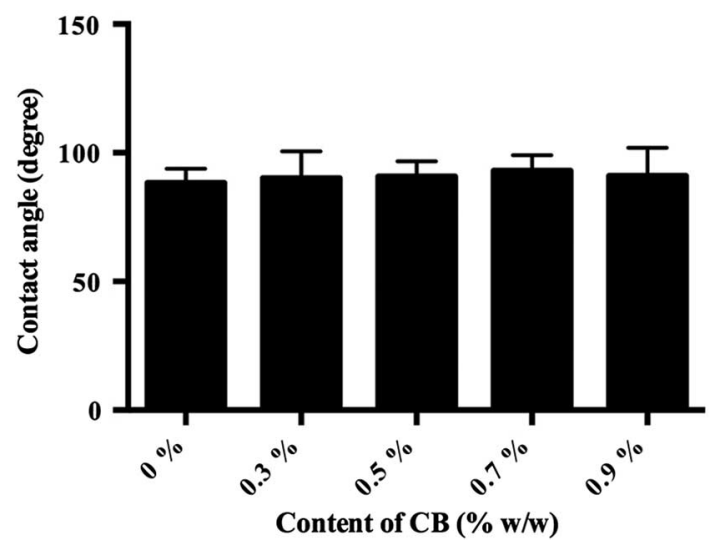

FIG. 10

FTIR-ATR spectra of the main components of the films under investigation.

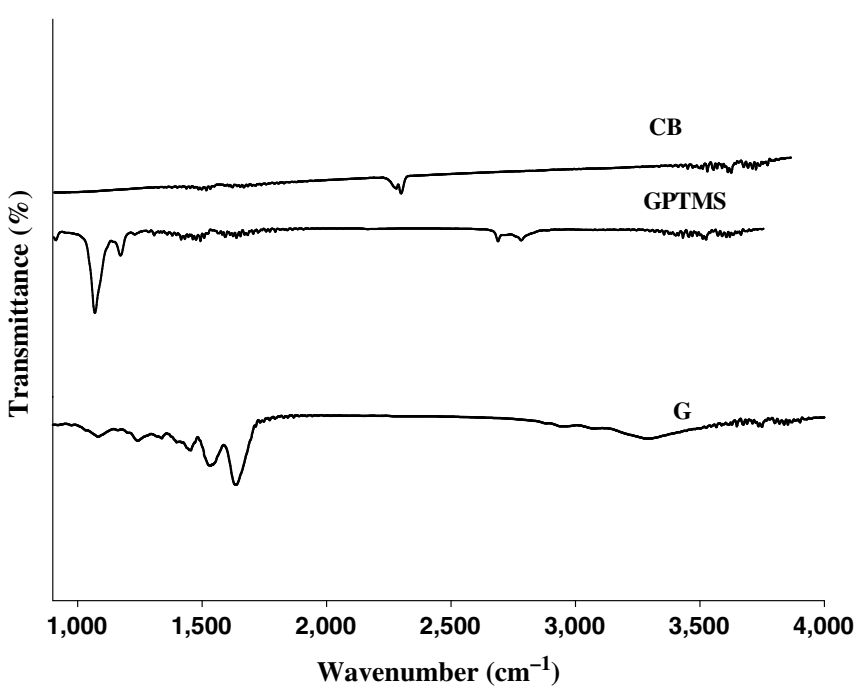

$920 \mathrm{~cm}^{-1}$ and $1,030 \mathrm{~cm}^{-1}$, respectively, as a demonstration of the formation of some cross-links (covalent bonds) between G and GPTMS. ${ }^{16}$ However, the spectra could not provide any information about the increasing quantities of $\mathrm{CB}$ within the specimens and about the efficacy of crosslinking process by increasing the $\mathrm{CB}$ concentration.

\section{IMPEDANCE MEASUREMENTS}

Besides physical, biochemical, or mechanical properties, the electrical cues are one of the main prerogatives that neural tissue constructs have to show to efficiently support the functionalities of neural cells. ${ }^{39}$ Therefore, impedance measurements were performed to investigate the electrical properties of round hydrated shaped gelatin-based films as a function of frequency for different incorporated quantity of CB. The results from impedance measurements are reported in figure 12. It was found that the impedance spectra of G-CB-GPTMS samples were consistent with those observed by others in the main neural tissues. ${ }^{40,41}$ As it can be observed in figure 8, pure cross-linked gelatin samples showed a higher electrical impedance as compared with composite ones in all the analyzed frequencies' spectrum. The impedance of the G-CB-GPTMS films exhibited a decreasing impedance trend with the increase in 
FIG. 11

FTIR-ATR spectra of the G-CB-GPTMS films for different $\mathrm{CB}$

concentrations.

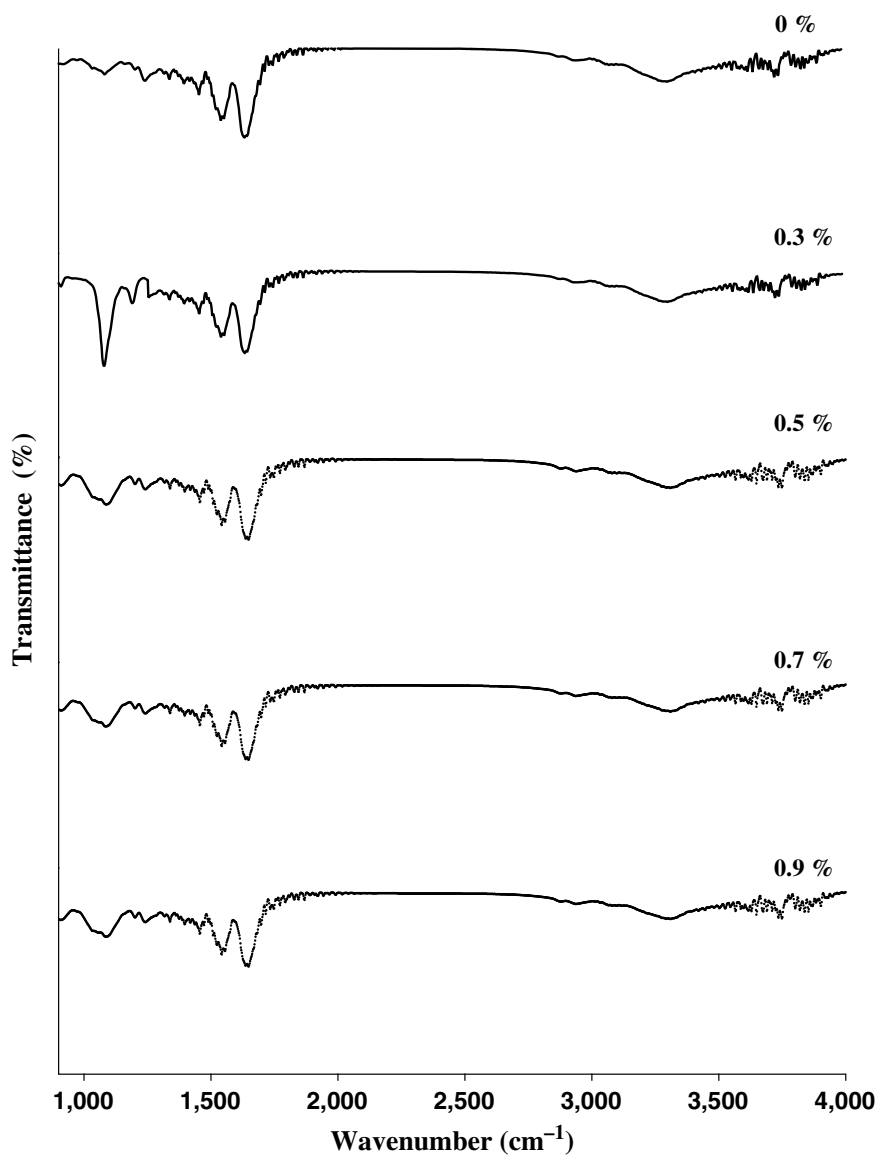

FIG. 12

\section{Electrical}

characterization.

Magnitude of electrical

impedance of G-CB-

GPTMS hydrogels as

a function of the

frequency for different

$\mathrm{CB}$ concentrations.

The measurements were performed after

hydrating the samples

for $2 \mathrm{~h}$ in Milli-Q ultrapure water $\left(25^{\circ} \mathrm{C}\right)$.

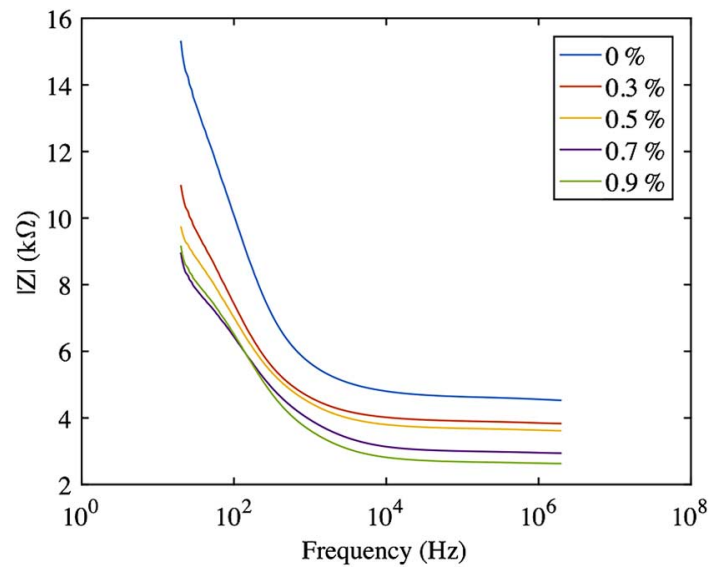

concentration of $\mathrm{CB}$ concentration; furthermore for each $\mathrm{CB}$ concentration, a significant decreasing of impedance was recorded around the frequencies commonly used in literature used for in vitro neural stimulations. ${ }^{42-44}$ This behavior clearly suggests that higher concentration of $\mathrm{CB}$ can favor the passage of an increasing resistive current 
through the $\mathrm{CB}$ network within the gelatin matrix, improving its electrical conductibility. The overall results demonstrated, on the one hand, the possibility to tune the electrical properties of the presented structures to control cell behaviors by providing proper electrical stimuli ${ }^{10}$; on the other hand, they demonstrated the possibility to employ the presented structures as sensing substrates to record impedance change correlated with cellular activity.

\section{Conclusions}

Recently, soft and biocompatible hydrogels with enhanced electrical properties have gained considerable attention in neural tissue engineering for their capability to enable not only biochemical and physical interactions but also electromechanical stimuli intimately mimicking the native neural tissues' ECM. In the presented study, chemically crosslinked and conductive hydrogels based on gelatin and different content of $\mathrm{CB}$ were fabricated in the form of films by the solvent casting technique and investigated for neural repair purpose. The proposed hydrogels were characterized in terms of morphological, compositional, swelling, dissolution, wettability, mechanical, and electrical properties as function of $\mathrm{CB}$ content. The films' thickness in hydrated state did not vary with the incremental content of $\mathrm{CB}$ reaching a value of $240.1 \pm 0.6 \mu \mathrm{m}$ (almost $70 \%$ more than that of dry films) after approximately $2 \mathrm{~h}$ of immersion in Milli-Q ultrapure water. The water uptake capability of the composite hydrogels relatively decreased for the $\mathrm{CB}$ concentration range of $0.3 \%-0.7 \%(w / w)$ in the gelatin matrix, while the dissolution in Milli-Q water significantly improved by increasing the percentage of $\mathrm{CB}$ content because it reduces the hydrogels' degradation over the time. In agreement with the degradation tests, the FTIR-ATR spectra on the G-CB-GPTMS revealed the occurrence of crosslinking process. It was even found that the well-known surface hydrophilicity of the pure gelatin did not change with the increase in CB content. From the mechanical point of view, the composite hydrogels exhibited a lower elastic modulus (7.7$9.3 \mathrm{MPa}$ ) with respect to that of pure cross-linked gelatin-based hydrogels $(15.49 \pm 0.64 \mathrm{MPa})$. Although the presented mechanical properties did not precisely match with those of the common neural tissue, nevertheless, this research evidence did not preclude the effectiveness of the presented structures for neural tissue regeneration as demonstrated by others in literature. ${ }^{35}$ The morphological characterization of the G-CB-GPTMS samples via SEM confirmed all the deduction about the mechanical and swelling properties of hydrogels. Further, SEM images demonstrated that the concentration of $0.9 \%$ of $\mathrm{CB}$ was too high to guarantee a uniform dispersion of the filling agent inside the gelatin matrix, causing a degradation of both mechanical and swelling properties.

Finally, the incorporation of an increasing content of $\mathrm{CB}$ enhanced the electrical performance of the gelatin hydrogel matrix as demonstrated by lowering the electrical impendence.

Based on the presented results, the proposed G-CB-GPTMS hydrogels could be considered as potential scaffolds with controllable and tunable mechanical, swelling, and electrical cues for tissue engineering applications. However, further studies are needed to assess the cells behavior on the presented structures and the effect of the electrical stimulation on cells adhesion and differentiation. On the basis of the cell tests' outcome, we could go on investigating if a modulation of the composition of the presented structures will be needed in order to get engineered hydrogels with improved mechanical, electrical, and morphological properties that better recapitulate those of the neural tissues.

\section{ACKNOWLEDGMENTS}

This work was supported by BIOMEMBRANE project (M-ERA.NET 2 project4246), by KERAPACK project (MANUNET MNET17/NMAT-0060), by PRA_2018_68 (grant supported by University of Pisa), and by IMAGO (MX18MO06) funded by MAECI and AMEXID.

\section{References}

1. X. Gu, "Progress and Perspectives of Neural Tissue Engineering," Frontiers of Medicine 9, no. 4 (December 2015): 401-411. https://doi.org/10.1007/s11684-015-0415-X

2. S. Song and P. M. George, "Conductive Polymer Scaffolds to Improve Neural Recovery," Neural Regeneration Research 12, no. 12 (December 2017): 1976-1978. https://doi.org/10.4103/1673-5374.221151 
3. A. Seddighi, A. Nikouei, A. S. Seddighi, A. R. Zali, S. M. Tabatabaei, A. R. Sheykhi, and S. Naeimian, "Peripheral Nerve Injury: A Review Article," International Clinical Neuroscience Journal 6, no. 1 (2016): 1-6. https://doi.org/10.1007/ s12013-013-9742-1

4. J. Ai, A. Kiasat-Dolatabadi, S. Ebrahimi-Barough, A. Ai, N. Lotfibakhshaiesh, A. Norouzi-Javidan, H. Saberi, B. Arjmand, and H. R. Aghayan, "Polymeric Scaffolds in Neural Tissue Engineering: A Review," Archives of Neuroscience 1, no. 1 (April 2014): 15-20. https://doi.org/10.5812/archneurosci.9144

5. X. Gu, F. Ding, and D. F. Williams, "Neural Tissue Engineering Options for Peripheral Nerve Regeneration," Biomaterials 35, no. 24 (August 2014): 6143-6156. https://doi.org/10.1016/j.biomaterials.2014.04.064

6. B. P. Chan and K. W. Leong, "Scaffolding in Tissue Engineering: General Approaches and Tissue-Specific Considerations," European Spine Journal 17, no. S4 (December 2008): 467-479. https://doi.org/10.1007/s00586-008-0745-3

7. S. Yang, L. K. Jang, S. Kim, J. Yang, K. Yang, S.-W. Cho, and J. Y. Lee, "Polypyrrole/Alginate Hybrid Hydrogels: Electrically Conductive and Soft Biomaterials for Human Mesenchymal Stem Cell Culture and Potential Neural Tissue Engineering Applications," Macromolecular Bioscience 16, no. 11 (July 2016): 1653-1661. https://doi.org/10. 1002/mabi.201600148

8. L. Ghasemi-Mobarakeh, M. P. Prabhakaran, M. Morshed, M. H. Nasr-Esfahani, H. Baharvand, S. Kiani, S. S. Al-Deyab, and S. Ramakrishna, "Application of Conductive Polymers, Scaffolds and Electrical Stimulation for Nerve Tissue Engineering," Journal of Tissue Engineering and Regenerative Medicine 5, no. 4 (January 2011): 17-35. https://doi. org/10.1002/term.383

9. X. Liu, K. J. Gilmore, S. E. Moulton, and G. G. Wallace, "Electrical Stimulation Promotes Nerve Cell Differentiation on Polypyrrole/Poly (2-Methoxy-5 Aniline Sulfonic Acid) Composites," Journal of Neural Engineering 6, no. 6 (December 2009): 065002. https://doi.org/10.1088/1741-2560/6/6/065002

10. G. M. Fortunato, C. De Maria, D. Eglin, T. Serra, and G. Vozzi, “An Ink-Jet Printed Electrical Stimulation Platform for Muscle Tissue Regeneration,” Bioprinting 11 (September 2018). https://doi.org/10.1016/j.bprint.2018.e00035

11. A. Subramanian, U. M. Krishnan, and S. Sethuraman, "Development of Biomaterial Scaffold for Nerve Tissue Engineering: Biomaterial Mediated Neural Regeneration,” Journal of Biomedical Science 16, no. 1 (November 2009): 108. https://doi.org/10.1186/1423-0127-16-108

12. D. Mawad, A. Lauto, and G. G. Wallace, "Conductive Polymer Hydrogels," in Polymeric Hydrogels as Smart Biomaterials, ed. S. Kalia (Berlin: Springer, 2016): 19-44.

13. D. Mawad, A. Artzy-Schnirman, J. Tonkin, J. Ramos, S. Inal, M. M. Mahat, N. Darwish, et al., "Electroconductive Hydrogel Based on Functional Poly(Ethylenedioxy Thiophene)," Chemistry of Materials 28, no. 17 (March 2016): 6080-6088. https://doi.org/10.1021/acs.chemmater.6b01298

14. Z. Shi, X. Gao, M. W. Ullah, S. Li, Q. Wang, and G. Yang, "Electroconductive Natural Polymer-Based Hydrogels," Biomaterials 111 (December 2016): 40-54. https://doi.org/10.1016/j.biomaterials.2016.09.020

15. S.-C. Wu, W.-H. Chang, G.-C. Dong, K.-Y. Chen, Y.-S. Chen, and C.-H. Yao, "Cell Adhesion and Proliferation Enhancement by Gelatin Nanofiber Scaffolds," Journal of Bioactive and Compatible Polymers 26, no. 6 (October 2011): 565-577. https://doi.org/10.1177/0883911511423563

16. C. Tonda-Turo, P. Gentile, S. Saracino, V. Chiono, V. K. Nandagiri, G. Muzio, R. A. Canuto, and G. Ciardelli, "Comparative Analysis of Gelatin Scaffolds Crosslinked by Genipin and Silane Coupling Agent," International Journal of Biological Macromolecules 49, no. 4 (November 2011): 700-706. https://doi.org/10.1016/j.ijbiomac.2011.07.002

17. C. Tonda-Turo, E. Cipriani, S. Gnavi, V. Chiono, C. Mattu, P. Gentile, I. Perroteau, M. Zanetti, and G. Ciardelli, "Crosslinked Gelatin Nanofibres: Preparation, Characterisation and In Vitro Studies Using Glial-Like Cells," Materials Science and Engineering: C 33, no. 5 (July 2013): 2723-2735. https://doi.org/10.1016/j.msec.2013.02.039

18. W. Phuchaduek, T. Jamnongkan, U. Rattanasak, S. Boonsang, and S. Kaewpirom, "Improvement in Physical and Electrical Properties of Poly(Vinyl Alcohol) Hydrogel Conductive Polymer Composites," Journal of Applied Polymer Science 132, no. 28 (April 2015): 42234. https://doi.org/10.1002/app.42234

19. D. Sahu, G. M. Kannan, and R. Vijayaraghavan, "Carbon Black Particle Exhibits Size Dependent Toxicity in Human Monocytes," International Journal of Inflammation 2014 (February 2014): 827019. https://doi.org/10.1155/2014/ 827019

20. M. Carrabba, C. De Maria, A. Oikawa, C. Reni, I. Rodriguez-Arabaolaza, H. Spencer, S. Slater, et al., "Design, Fabrication and Perivascular Implantation of Bioactive Scaffolds Engineered with Human Adventitial Progenitor Cells for Stimulation of Arteriogenesis in Peripheral Ischemia," Biofabrication 8, no. 1 (March 2016): 015020. https://doi.org/ 10.1088/1758-5090/8/1/015020

21. S. Farris, K. M. Schaich, L. Liu, P. H. Cooke, L. Piergiovanni, and K. L. Yam, "Gelatin-Pectin Composite Films from Polyion-Complex Hydrogels," Food Hydrocolloids 25, no. 1 (January 2011): 61-70. https://doi.org/10.1016/j.foodhyd. 2010.05.006

22. M.-W. Yang and S.-Y. Lin, “A Method for Correcting the Contact Angle from the $\theta / 2$ Method," Colloids and Surfaces A: Physicochemical and Engineering Aspects 220, nos. 1-3 (June 2003): 199-210. https://doi.org/10.1016/S0927-7757(03)00064-5

23. H. Baniasadi, R. S. A. Ramazani, and M. Shohreh, "Fabrication and Characterization of Conductive Chitosan/ Gelatin-Based Scaffolds for Nerve Tissue Engineering," International Journal of Biological Macromolecules 74 (March 2105): 360-366. https://doi.org/10.1016/j.ijbiomac.2014.12.014

24. A. L. Daniel-da-Silva, A. M. Salgueiro, and T. Trindade, "Effects of Au Nanoparticles on Thermoresponsive GenipinCrosslinked Gelatin Hydrogels,” Gold Bulletin 46, no. 1 (March 2013): 25-33. https://doi.org/10.1007/s13404-012-0078-1 
25. G. Kavoosi, S. M. M. Dadfar, S. M. A. Dadfar, F. Ahmadi, and M. Niakosari, "Investigation of Gelatin/Multi-Walled Carbon Nanotube Nanocomposite Films as Packaging Materials," Food Science \& Nutrition 2, no. 1 (December 2014): 65-73. https://doi.org/10.1002/fsn3.81

26. M. L. K. Tate, "Whither Flows the Fluid in Bone? An Osteocyte's Perspective," Journal of Biomechanics 36, no. 10 (October 2003): 1409-1424. https://doi.org/10.1016/S0021-9290(03)00123-4

27. S. R Hynes, M. F. Rauch, J. P. Bertram, and E. B. Lavik, “A Library of Tunable Poly(Ethylene Glycol)/Poly (L-Lysine) Hydrogels to Investigate the Material Cues that Influence Neural Stem Cell Differentiation," Journal of Biomedical Materials Research Part A 89, no. 2 (May 2009): 499-509. https://doi.org/10.1002/jbm.a.31987

28. J. R. Soucy, E. S. Sani, R. P. Lara, D. Diaz, F. Dias, A. S. Weiss, A. N. Koppes, R. A. Koppes, and N. Annabi, "Photocrosslinkable Gelatin/Tropoelastin Hydrogel Adhesives for Peripheral Nerve Repair," Tissue Engineering Part A 24, nos. 17-18 (September 2018): 1393-1405. https://doi.org/10.1089/ten.tea.2017.0502

29. N. Al Habis, A. El Moumen, M. Tarfaoui, and K. Lafdi, "Mechanical Properties of Carbon Black/Poly ( $\varepsilon$-Caprolactone)Based Tissue Scaffolds," Arabian Journal of Chemistry (in press) (2018). https://doi.org/10.1016/j.arabjc.2018.10.005

30. R. S. H. Wong, M. Ashton, and K. Dodou, "Effect of Crosslinking Agent Concentration on the Properties of Unmedicated Hydrogels," Pharmaceutics 7, no. 3 (September 2015): 305-319. https://doi.org/10.3390/pharmaceutics7030305

31. G. Kavoosi, S. M. M. Dadfar, S. M. A. Dadfar, F. Ahmadi, and M. Niakosari, "Investigation of Gelatin/Multi-Walled Carbon Nanotube Nanocomposite Films as Packaging Materials," Food Science \& Nutrition 2, no. 1 (December 2014): 65-73. https://doi.org/10.1002/fsn3.81

32. H. Li, D. Q. Wang, H. L. Chen, B. L. Liu, and L. Z. Gao, "A Novel Gelatin-Carbon Nanotubes Hybrid Hydrogel," Macromolecular Bioscience 3, no. 12 (December 2003): 720-724. https://doi.org/10.1002/mabi.200300034

33. Y. Whulanza, E. Battini, L. Vannozzi, M. Vomero, A. Ahluwalia, and G. Vozzi, "Electrical and Mechanical Characterization of Single Wall Carbon Nanotubes Based Composites for Tissue Engineering Applications," Journal of Nanoscience and Nanotechnology 13, no. 1 (January 2013): 188-197. https://doi.org/10.1166/jnn.2013.6708

34. L. Ghasemi-Mobarakeh, M. P. Prabhakaran, L. Tian, E. Shamirzaei-Jeshvaghani, L. Dehghani, and S. Ramakrishna, "Structural Properties of Scaffolds: Crucial Parameters Towards Stem Cells Differentiation," World Journal of Stem Cells 7, no. 4 (May 2015): 728-744. https://doi.org/10.4252/wjsc.v7.i4.728

35. M. Jafarkhani, Z. Salehi, and T. Nematian, "Preparation and Characterization of Chitosan/Graphene Oxide Composite Hydrogels for Nerve Tissue Engineering" (paper presentation, INN International Conference/Workshop on Nanotechnology and Nanomedicine, Tehran, Iran, May 2-3, 2017).

36. K. L. Menzies and L. Jones, "The Impact of Contact Angle on the Biocompatibility of Biomaterials," Optometry and Vision Science 87, no. 6 (June 2010): 387-399. https://doi.org/10.1097/OPX.0b013e3181da863e

37. J. Yuan, S. Zhou, G. Gu, and L. Wu, "Effect of the Particle Size of Nanosilica on the Performance of Epoxy/Silica Composite Coatings," Journal of Materials Science 40, no. 15 (August 2015): 3927-3932. https://doi.org/10.1007/ s10853-005-0714-8

38. J. H. Muyonga, C. G. B. Cole, and K. G. Duodu, "Fourier Transform Infrared (FTIR) Spectroscopic Study of Acid Soluble Collagen and Gelatin from Skins and Bones of Young and Adult Nile Perch (Lates niloticus)," Food Chemistry 86, no. 3 (July 2004): 325-332. https://doi.org/10.1016/j.foodchem.2003.09.038

39. J. H. Min, M. Patel, and W.-G. Koh, "Incorporation of Conductive Materials into Hydrogels for Tissue Engineering Applications,” Polymers 10, no. 10 (2018): 1-36. https://doi.org/10.3390/polym10101078

40. J. Rozman, M. C. Žužek, R. Frangež, and S. Ribarič, "Measurement of Bio-Impedance on an Isolated Rat Sciatic Nerve Obtained with Specific Current Stimulating Pulses," Materials and Technology 50, no. 5 (October 2016): 797-804. https:// doi.org/10.17222/mit.2015.307

41. P. N. Robillard and Y. Poussart, "Specific-Impedance Measurements of Brain Tissues," Medical and Biological Engineering and Computing 15, no. 4 (July 1977): 438-445. https://doi.org/10.1007/BF02457999

42. J. Huang, L. Lu, J. Zhang, X. Hu, Y. Zhang, W. Liang, S. Wu, and Z. Luo, "Electrical Stimulation to Conductive Scaffold Promotes Axonal Regeneration and Remyelination in a Rat Model of Large Nerve Defect," PLoS ONE 7, no. 6 (June 2012): e39526. https://doi.org/10.1371/journal.pone.0039526

43. M. Hronik-Tupaj, W. K. Raja, M. Tang-Schomer, F. G. Omenetto, and D. L. Kaplan, "Neural Responses to Electrical Stimulation on Patterned Silk Film," Journal of Biomedical Materials Research Part A 101, no. 9 (July 2013): 2559-2572. https://doi.org/10.1002/jbm.a.34565

44. R. Balint, N. J. Cassidy, and S. H. Cartmell, "Electrical Stimulation: A Novel Tool for Tissue Engineering," Tissue Engineering Part B: Reviews 19, no. 1 (February 2013): 48-57. https://doi.org/10.1089/ten.TEB.2012.0183 